\title{
Complicaciones de traqueostomía en pacientes pediátricos. Revisión de la literatura
}

\author{
Complications of tracheostomy in pediatric patients. Literature review \\ Hiram Álvarez N¹, Perla Villamor¹.
}

\begin{abstract}
RESUMEN
Las indicaciones de traqueostomía en niños han cambiado considerablemente en los últimos años, así como el perfil epidemiológico de los pacientes y la morbimortalidad de este procedimiento. Las complicaciones de este procedimiento pueden ser clasificadas en intraoperatorias, inmediatas y tardías. La mortalidad global en pacientes pediátricos llevados a traqueostomía oscila entre el 13\% y 19\%. Sin embargo, menos del $5 \%$ es directamente atribuible a la cirugía. La presente revisión abarcará las complicaciones más frecuentes asociadas a traqueostomía pediátrica con recomendaciones en su prevención y manejo.
\end{abstract}

Palabras clave: Traqueostomía pediátrica, vía aérea pediátrica, complicaciones quirúrgicas.

\section{ABSTRACT}

Indications of tracheostomy in children have changed considerably in recent years, as well as the epidemiological profile of patients, and morbidity and mortality related to this procedure. Complications of pediatric tracheostomy can be classified into intraoperative, immediate and late. Overall mortality in pediatric patients with tracheostomy ranges from $13 \%$ to $19 \%$. However, less than $5 \%$ is directly attributable to tracheostomy. This review will cover the most frequent complications associated with pediatric tracheostomy with recommendations for its prevention and management.

Key words: Pediatric tracheostomy, pediatric airway, surgical complications.

\section{INTRODUCCIÓN}

Las indicaciones de traqueostomía en niños han cambiado considerablemente en los últimos años, en comparación con la población adulta donde las indicaciones han permanecido similares ${ }^{1}$. Previamente, la principal indicación eran las infecciones. Sin embargo, con los avances en la medicina neonatal e inmunizaciones, la indicación infecciosa ha disminuido y las causas

Otorrinolaringología Pediátrica. Hospital Infantil de México Federico Gómez, Universidad Nacional Autónoma de México. 
neurológicas y el soporte ventilatorio prolongado son indicaciones más frecuentes cada día $a^{1,2}$.

Así como las indicaciones de traqueostomía y el perfil epidemiológico de los pacientes pediátricos que requieren este procedimiento han experimentado cambios importantes en las últimas décadas, la morbimortalidad y sus complicaciones relacionadas también se han modificado notablemente $^{3}$. Adicionalmente, numerosos autores han destacado que las complicaciones son más frecuentes en niños que en adultos ${ }^{3-5}$, ya que a menudo cuentan con múltiples factores predisponentes y comorbilidades médicas, sumado a la vulnerabilidad asociada a las características anatómicas y fisiológicas propias del niño ${ }^{6}$.

La traqueostomía en población pediátrica requiere una especial atención y cuidados en el posoperatorio inmediato, así como cuidados a largo plazo. La educación de los padres y cuidadores en cuanto al manejo posoperatorio adecuado es fundamental para reducir las complicaciones y mortalidad después de esta cirugía 4 .

\section{MORBIMORTALIDAD ASOCIADA}

Se ha estimado una tasa de complicaciones entre $10 \%$ y $58 \%$, con una mortalidad directamente relacionada menor al 5\%3,7. De acuerdo a una revisión sistemática de la literatura recientemente publicada, que incluyó 48 estudios rigurosamente seleccionados, la mortalidad asociada directamente a una traqueostomía tiene un rango de 0\% a 5,9\% entre diferentes investigadores. Con base a estos reportes, la mortalidad ha variado de $2,9 \%$ en la década de 1985 a 1994 a 0,9\% entre 2005 a $2014^{3}$. Sin embargo, las complicaciones relacionadas siguen siendo elevadas y pueden ser clasificadas en intraoperatorias, cuando suceden durante la cirugía propiamente dicha; inmediatas, cuando se presentan dentro de la primera semana de evolución; y tardías, cuando se presentan semanas 0 meses después de realizado el procedimiento ${ }^{3}$ (Tabla 1).

Estudios de cohortes han demostrado que la enfermedad broncopulmonar, la cardiopatía congénita, la infección sistémica severa y las neoplasias, son factores de riesgo independientes para mortalidad ${ }^{8,9}$. Por otra parte, edades inferiores a los 2 años también se han demostrado como un factor de riesgo independiente ${ }^{10}$. En cuanto a la mortalidad directamente atribuible a la traqueostomía, se han encontrado dos principales causas: la remoción accidental de la cánula y la obstrucción de la misma $^{11,12}$. Las tasas de morbilidad y mortalidad dependen significativamente de lo bien informado y capacitado que esté el equipo médico, así como los padres y cuidadores de los pacientes 5 . Para evitar complicaciones accidentales y mortales, es necesario proporcionar una instrucción suficiente en cuanto al manejo de la cánula tanto en el ambiente hospitalario como en casa. Para ello, tanto profesionales de la salud a cargo del menor, como principales cuidadores deben poseer conocimientos básicos en resucitación cardiopulmonar, ventilación manual con máscara, intercambio de cánulas traqueales y succión de las vías respiratorias ${ }^{4,8}$.

\subsection{Complicaciones intraoperatorias}

Las lesiones intraoperatorias son consecuencia directa de una evaluación y/o técnica operatoria inadecuada. Hoy en día, las indicaciones de

Tabla 1. Complicaciones de traqueostomía

\begin{tabular}{|lll|}
\hline Intraoperatorias & Tempranas & Tardías \\
\hline Lesiones vasculares & Obstrucción de la cánula & Granuloma \\
Neumotórax y neumomediastino & Decanulación accidental & Estenosis subglótica \\
Atelectasia & Falsa vía & Traqueomalacia segmentaria \\
Lesión de nervios laríngeos recurrentes & Neumotórax & Colapso supraestomal \\
Lesión esofágica & Hipoxia & Fístula traqueocutánea posdecanulación \\
& Hemorragia erosiva & \\
& Infección y exposición del estoma & \\
\hline
\end{tabular}


traqueostomía son cada vez más del orden de la cirugía electiva. Los procedimientos urgentes realizados en condiciones y/o equipo inadecuados solo incrementan la posibilidad de complicaciones, que incluso pueden ser fatales ${ }^{3}$. Por ello, el mejor lugar para llevar a cabo esta cirugía es en un quirófano, con el instrumental y equipo completo, con una colocación adecuada del paciente, así como la participación de un anestesiólogo experto en vía aérea pediátrica, particularmente importante en los lactantes y niños pequeños donde el cirujano puede perderse en el campo operatorio por tener una experiencia limitada.

\subsubsection{Lesiones vasculares}

La mayoría de las complicaciones intraoperatorias se deben a lesiones de tejidos adyacentes a la tráquea 0 en la inmediación del campo quirúrgico, que en niños pequeños puede ser muy reducido. Las lesiones vasculares se presentan en menos del $1 \%$. Sin embargo, en el caso de lesionarse vasos importantes como arterias 0 venas tiroideas, yugulares, carótidas 0 incluso del arco braquiocefálico, se pueden desencadenar eventos hemorrágicos fatales ${ }^{13}$. La lesión de la arteria innominada puede ser causada por la creación del estoma a un nivel inferior al cuarto anillo traqueal. Las tráqueas de niños y adolescentes son móviles y se elevan en el cuello junto con la arteria innominada en hiperextensión. Una arteria innominada más alta de lo normal en relación con la tráquea se ha reportado hasta en el $12 \%$ de los $\operatorname{casos}^{13}$. La lesión vascular puede presentarse durante el acto quirúrgico, así como en el posoperatorio resultado de una erosión de la arteria innominada por el contacto directo con la cánula de traqueostomía. Es importante recordar que recién nacidos y lactantes tienen un volumen intravascular promedio de 70 a $80 \mathrm{ml} / \mathrm{kg}$ de peso. Ejemplificando, un niño de 4 kilogramos tendría $280 \mathrm{ml}$ de sangre total por lo que un sangrado de 14 mililitros sería $5 \%$ de su volumen sanguíneo ${ }^{14}$. En el caso de presentarse una lesión vascular intraoperatoria, el manejo principal consiste en la reparación de la lesión en el mismo tiempo quirúrgico. Sin embargo, si se produce una hemorragia masiva en el posoperatorio, el manejo implicará tres prioridades simultáneas: el mantenimiento de una vía respiratoria permeable, el control de la hemorragia y la reanimación cardiopulmonar básica ${ }^{13}$.

\subsubsection{Neumotórax}

Otra lesión probable es la creación de una falsa vía, con neumotórax y extensión al mediastino. En el paciente pediátrico las cúpulas pleurales pueden encontrarse muy altas y alcanzar la base del cuello, lo que hace posible este tipo de lesiones si el cirujano no mantiene la disección estrictamente en la línea media 5 . Del mismo modo, la tráquea es mucho más superficial, delgada y fina, lo que puede condicionar una lesión perforante, e incluso lacerante durante la disección. En décadas previas ésta era una de las complicaciones más frecuentes, hoy en día, su incidencia ha disminuido gracias a la norma de realizar el procedimiento en un quirófano con el paciente en posición adecuada ${ }^{5}$. El deterioro súbito de un paciente con desaturación grave y bradicardia durante la realización de una traqueostomía puede deberse a un neumotórax 0 un neumomediastino. La punción-aspiración puede ser obligada para estabilizar al paciente.

Se puede realizar una radiografía posoperatoria para excluir la presencia de un neumotórax que pudo resultar de una lesión en el ápice pulmonar 0 una ventilación de alta presión en neonatos. Aunque se ha argumentado que no debe ser rutinaria, sino que debe reservarse para los casos en los que haya motivos para sospechar una complicación ${ }^{15}$.

\subsubsection{Otras lesiones intraoperatorias}

Es importante considerar que las pequeñas estructuras del niño pueden perderse de la línea media fácilmente, su lateralización puede ser secundario al uso inadecuado de los separadores por parte de un ayudante inexperto. Lo anterior, facilita las lesiones esofágicas e incluso vertebrales al profundizarse la búsqueda de una tráquea que no se logra ubicar por este motivo. La disección lateral y excesiva de la tráquea puede dar lugar a una lesión directa de los nervios laríngeos recurrentes que puede derivar en una parálisis transitoria 0 permanente de las cuerdas vocales.

\subsection{Complicaciones posoperatorias tempranas}

El periodo de máximo riesgo de una traqueostomía, comprende los primeros 7 a 8 días de realizada la cirugía. En este periodo aún no se ha formado 0 madurado el estoma propiamente dicho. Durante este lapso no debería retirarse la cánula de 
traqueostomía. Puede ser imposible recolocarla limpiamente, dando lugar a creación de una falsa vía, neumotórax, enfisema masivo o un evento asfíctico con consecuencias fatales.

\subsubsection{Cuidados generales durante la primera semana de posoperatorio}

Idealmente el paciente recién intervenido debería permanecer en una sala de cuidados intermedios, donde pueda ser monitorizado los primeros días, y cuyo personal asignado a sus cuidados conozca los procedimientos requeridos en caso de una complicación temprana y la serie de acciones en caso de decanulación accidental. El personal debe estar informado acerca de el número y marca de la cánula utilizada, diámetro interno y su longitud, la causa original de la traqueostomía, si hay una condición obstructiva a nivel laríngeo 0 de la tráquea proximal, si el paciente utiliza o no un stent en la laringe y las condiciones médicas especiales de cada paciente. Esta información debe estar a la vista en un cartel a la cabecera del paciente. La sala de cuidados también debe contar con un box 0 kit de decanulación en caso de extrusión de la cánula y disponer de equipo de resucitación, oxígeno continuo y aspiración de pared. La rutina de cuidados debe incluir un calendario y reporte de aspiraciones, carteles informativos (acciones en obstrucción de la cánula, decanulación accidental y técnica de aspiración a dos manos), así como manual de procedimientos. El personal de una clínica de cuidado de estomas debe formar parte de los cuidados de estos pacientes cuando se requiera de su participación en caso de infección 0 exposición del estoma.

\subsubsection{Obstrucción de la cánula}

La complicación temprana más común de una traqueostomía en un paciente pediátrico es la obstrucción de la cánula ${ }^{11}$. La obstrucción tiene un amplio rango de causas, siendo la más común la presencia de secreciones o coágulos de sangre en la cánula. La aspiración, humidificación y limpieza de la cánula interna, tanto inmediatamente después de la operación como después del período posoperatorio inmediato (24-72 horas) evita la obstrucción del tubo. Una cánula que produce sonido, puede estar parcialmente obstruida. Esto puede acompañarse de inquietud e incomodidad en el paciente, taquicardia leve, esfuerzo respiratorio y desaturaciones. Una cánula completamente obstruida no hace ruido y el paciente tiene grandes esfuerzos respiratorios, cianosis y taquicardia. En el paciente con una traqueostomía reciente no debe retirarse la cánula a menos que haya fracaso respiratorio inminente, ya que la recolocación de otra cánula puede ser imposible. Si hay un bloqueo importante deben fluidificarse con solución salina tibia las secreciones que obstruyen la cánula y aspirarse profusamente, si esto no resuelve el bloqueo, se puede pasar completa la guía de la cánula a través de la misma, que debe estar siempre a la mano para empujar un probable tapón bien consolidado. Si la cánula tiene al menos seis días de realizada, la opción sería retirar la cánula y cambiarla por otra nueva. Ante la presencia de desaturación y preocupación de una posible obstrucción sin evidencia de un tapón de moco 0 presencia de un coágulo sanguíneo, es importante visualizar la posición del tubo en la tráquea del paciente con el uso de un laringoscopio flexible para asegurar la adecuada posición y permeabilidad del mismo ${ }^{16}$.

\subsubsection{Decanulación accidental}

La decanulación accidental puede resultar en una morbilidad significativa e incluso mortalidad cuando se produce en pacientes con una traqueostomía reciente ( $<7$ días). Es la complicación más temida y puede dar lugar a una serie de eventos rápidamente sucesivos: desaturación súbita, retención de $\mathrm{CO}_{2}$, hipoxia, acidosis respiratoria, paro cardiopulmonar, lesión posasfíctica y/o muerte. Ésta generalmente pasa inadvertida y es más frecuente después de maniobras de movilización del paciente inadecuadas, que realiza una sola persona sin ayuda, tales como aspiraciones, toma de productos 0 estudios de imagen ${ }^{17}$.

El personal de guarda debe considerar la gravedad de este evento y anticipar la imposibilidad de restablecer una vía aérea exitosa y prontamente. Debe ser manejado en equipo como un evento de paro cardiopulmonar, sin tiempos muertos y actuar secuencialmente. Los pacientes con patologías respiratorias importantes, con aumento de la circunferencia del cuello, oxigenación marginal 0 en los que el tubo de traqueostomía es esencial para la ventilación mecánica parecen ser particularmen- 
te vulnerables ${ }^{17}$. En estas situaciones, la permeabilidad de las vías respiratorias debe restablecerse rápidamente para evitar la hipoxemia prolongada, lesiones orgánicas o muerte. Sin embargo, si la reinserción de la cánula no se realiza cuidadosamente, se puede formar una falsa vía anterior a la tráquea durante la reinserción del tubo. Tan pronto como se reinserta la cánula de traqueostomía, la colocación debe ser confirmada mediante auscultación, observación de la elevación del tórax y documentación de oximetría y capnografía. Además, se debe vigilar el desarrollo de enfisema subcutáneo en el cuello, cuya aparición podría indicar la colocación del tubo en un tracto falso de los tejidos blandos o una perforación traqueal.

Para prevenir la remoción accidental de la cánula, se debe ajustar cuidadosamente la cinta, colocando suturas de seguridad no absorbibles a través de la tráquea a cada lado de la incisión traqueal. Ante una decanulación accidental, las suturas se pueden retraer hacia ambos lados para abrir la incisión y traer la tráquea a la superficie, logrando una reinserción segura y eficaz de la cánula. Las suturas o "riendas" de seguridad adecuadamente colocadas son la mejor forma de prevenir una falsa ruta después de una decanulación no programada (Figura 1).

\subsubsection{Otras complicaciones del posoperatorio inmediato}

Las lesiones vasculares, ya comentadas previamente, pueden presentarse mediatamente por erosión vascular varios días después de realizada la traqueostomía. Aunque son verdaderamente raras, son eventos catastróficos que ponen en peligro la vida. Otras adversidades son las relativas a la exposición de segmentos traqueales por infección local y presión sobre el área del estoma (Figura 2). El manejo incluye curación diaria y aplicación tópica

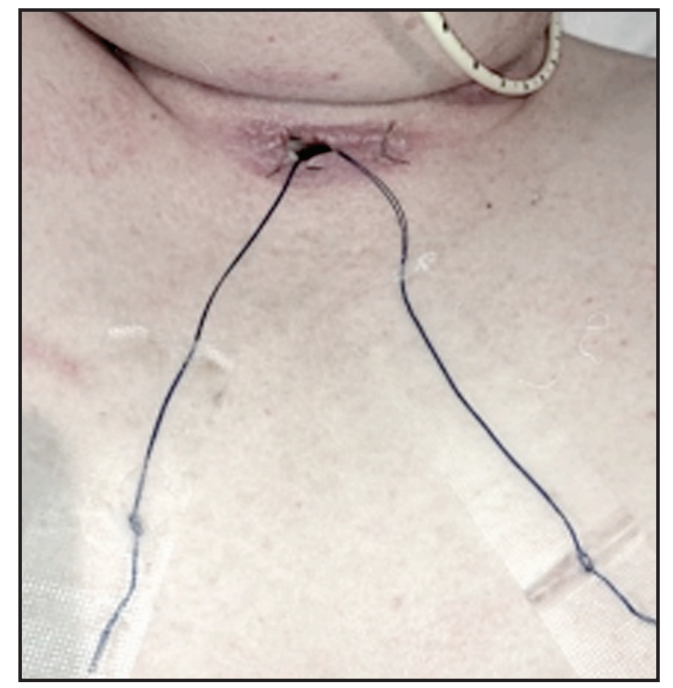

Figura 1. Riendas en estoma de traqueostomía.

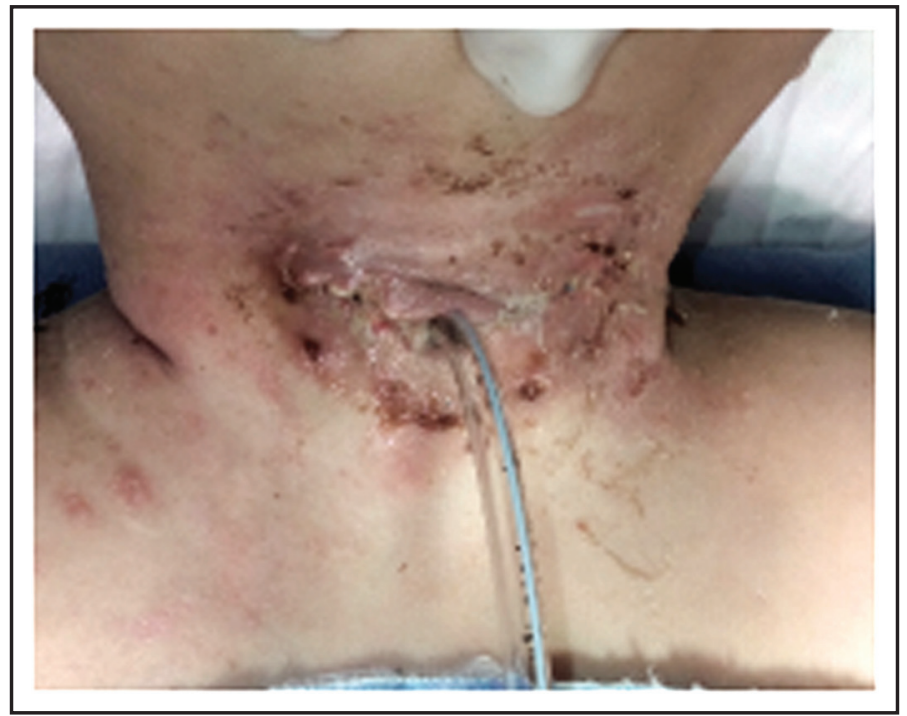

Figura 2. Infección local con exposición de segmento traqueal. 
de antibióticos en ungüento y favorecedores de la cicatrización, en ocasiones puede ser necesario el cierre por segunda intención.

\subsection{Complicaciones tardías}

Entre ellas se incluyen: granuloma supraestomal, estenosis subglótica, traqueomalacia, erosión de la carina y fistula traqueocutánea persistente una vez retirada la cánula. La mayoría de estas complicaciones se relacionan a un tamaño inadecuado de la cánula, por lo que la selección de la cánula es muy importante. La selección apropiada de la cánula de traqueostomía permite mantener una ventilación adecuada al mismo tiempo que previene complicaciones. La cánula debe ser lo suficientemente pequeña para no lesionar la mucosa traqueal, evitando la aparición de estenosis o granulomas, y debe ser lo suficientemente grande para evitar la hipoventilación o la extubación

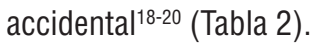

\subsubsection{Granuloma supraestomal}

Los granulomas son la complicación tardía más frecuente de una traqueostomía, la incidencia de los granulomas de la pared traqueal varía del $4 \%$ al $80 \%{ }^{21,22}$. Los granulomas con mayor frecuencia son supraestomales pero también pueden ocurrir en la punta inferior de la cánula si ésta es demasiado larga. Los granulomas supraestomales son consecuencia de una irritación e infección de la mucosa por la cánula de traqueostomía. Con frecuencia no dan problemas y son lesiones blandas y friables fácilmente resecables. No obstante, en ocasiones se acompañan de fibrosis marcada y neoformación cartilaginosa por condritis crónica, en los márgenes del traqueostoma con una obstrucción importante de la luz respiratoria que impide la decanulación del paciente a pesar de haberse resuelto la causa original y motivo de la traqueostomía. Con frecuencia son de difícil manejo y deben ser retirados quirúrgicamente por cirugía endoscópica a través de una laringoscopía de suspensión.

\subsubsection{Estenosis subglótica}

Esta es una complicación cada vez menos frecuente de origen iatrogénico por una traqueostomía demasiado alta en la cercanía de los bordes del cartílago cricoides donde la curvatura de la cánula condiciona una fricción constante que deriva en formación de granuloma, fibrosis y finalmente la estenosisist,23. El uso de fibroscopios para asegurarse de la posición de la cánula evita esta complicación. Aunque es poco frecuente, es la primera causa de estenosis traqueal en niños. Una vez establecida la lesión, la única manera de tratarla es con cirugía reconstructiva.

\subsubsection{Traqueomalacia segmentaria}

El uso prolongado de una traqueostomía se asocia a la formación de traqueomalacia. La pérdida de soporte cartilaginoso por absorción de anillos traqueales puede terminar en malacia segmentaria. Aunque no exista una deformidad aparente u obstrucción mecánica, al retirar la cánula puede presentarse un colapso con los movimientos inspiratorios, generando una obstrucción dinámica que obliga a mantener la cánula. Desafortunadamente no existe algún tipo de manejo definitivo para la traqueomalacia ${ }^{21}$, aunque se han intentado la suspensión traqueal y el uso de prótesis externas como miniplacas.

Tabla 2. Tamaño cánula de traqueostomía por edad y peso

\begin{tabular}{|c|c|c|c|}
\hline Edad & Peso (Kg) & Tamaño (mm) & Largo (cm) \\
\hline Neonato & $2-4$ & $2,5-3,5$ & $10-12$ \\
\hline $1-6$ meses & $4-6$ & $4,0-4,5$ & $12-14$ \\
\hline 6-12 meses & $6-10$ & $4,5-5,0$ & $14-16$ \\
\hline 1-3 años & $10-15$ & $5,0-5,5$ & $16-18$ \\
\hline 4-6 años & $15-20$ & $5,5-6,5$ & $18-20$ \\
\hline 7-10 años & $25-35$ & $6,5-7,0$ & $20-22$ \\
\hline 10-14 años & $40-50$ & $7,0-7,5$ & $22-24$ \\
\hline
\end{tabular}




\subsubsection{Colapso supraestomal}

Otra causa de fracaso en la decanulación de un paciente pediátrico traqueostomizado es el colapso o malacia supraestomal, la cual representa una obstrucción de la vía aérea proximal a la traqueostomía. Aunque ocasiona una importante disminución de la luz traqueal, no se trata de una auténtica estenosis ya que no es una lesión rígida y permite el paso del fibrobroncoscopio sin dificultad. Se ha descrito una mayor incidencia en pacientes traqueostomizados con una técnica de ventana traqueal con colgajos laterales, en comparación con la técnica de traqueofisura vertical anterior. El colapso habitualmente es leve y no interfiere con la decanulación del paciente. Sin embargo, en aproximadamente $10 \%$ de las traqueostomías de más de un año de evolución, el cuadro obstructivo es grave y la decanulación no es factible, requiriendo corrección quirúrgica ${ }^{24}$.

\section{BIBLIOGRAFÍA}

1. Douglas CM, Poole-Cowley J, Morrissey S, Kubba H, Clement WA, Wynne D. Paediatric tracheostomy-An 11 year experience at a Scottish paediatric tertiary referral centre. Int J Pediatr Otorhinolaryngol 2015; 79: 1673-6.

2. Ogilvie LN, Kozak JK, Chiu S, Adderley RJ, Kozak FK. Changes in pediatric tracheostomy 19822011: a Canadian tertiary children's hospital review. J Pediatr Surg 2014; 49: 1549-53.

3. Campisi P, Forte V. Pediatric tracheostomy. Semin Pediatr Surg 2016; 25: 191-5.

4. Gaudreau PA, Greenlick H, Dong T, Levy M, Hackett A, Preciado D, Zalzal G, Rellly BK. Preventing Complications of Pediatric Tracheostomy Through Standardized Wound Care and Parent Education. JAMA Otolaryngol Head Neck Surg 2016; 142: 966-71.

5. Dal'Astra AP, Quirino AV, Caixêta JA, Avelino MA. Tracheostomy in childhood: review of the literature on complications and mortality over the last three decades. Braz J Otorhinolaryngol 2017; 83: 207-14.

6. Ha JF, Ostwani W, Green G. Successful conservative management of a rare complication of

\subsubsection{Fístula traqueocutánea persistente}

La fístula traqueocutánea, es una complicación tardía. En la mayoría de los casos, el estoma cierra por segunda intención al retirar la cánula. Sin embargo, entre el $1 \%$ y $3 \%$ de los pacientes, después de la decanulación, pueden desarrollar una fístula persistente secundaria a la epitelización escamosa del estoma. Una serie de 172 pacientes, no evidenció una diferencia estadísticamente significativa en la incidencia de fístula traqueocutánea ni formación de tejido de granulación entre estomas a los que se les realizó sutura de la piel a los bordes del cartílago traqueal (maduración quirúrgica) y los que no tenían suturas $^{26}$. Por otra parte, la incidencia de fístula traqueocutánea sí está asociada a traqueostomías de larga evolución ${ }^{26}$. La fístula puede resultar en secreciones persistentes con irritación de la piel, fonación perturbada, infecciones frecuentes y alteración cosmética. En cuyo caso estaría indicado el manejo quirúrgico ${ }^{26,27}$.

tracheostomy; extensive posterior tracheal false pouch. Int J Pediatr Otorhinolaryngol 2016; 90: 54-7.

7. Carr MM, Poje CP, Kingston L, Kielma D, Heard C. Complications in pediatric tracheostomies. Laryngoscope 2001; 111: 1925-8.

8. Funamura Jl, Yuen S, Kawai K, Gergin 0, Adil E, Rahbar R, Watters K. Characterizing mortality in pediatric tracheostomy patients. Laryngoscope 2017; 127: 1701-6.

9. Lee JH, Smith PB, Quek MB, Laughon MM, Clark RH, HoRNIK CP. Risk Factors and In-Hospital Outcomes following Tracheostomy in Infants. $J$ Pediatr 2016; 173: 39-44.

10. Mahida JB, Asti L, Boss EF, Shah RK, Deans KJ, Minneci PC, Jatana KR. Tracheostomy Placement in Children Younger Than 2 Years: 30-Day Outcomes Using the National Surgical Quality Improvement Program Pediatric. JAMA Otolaryngol Head Neck Surg 2016; 142: 241-6.

11. Tsubol N, Ide K, Nishimura N, Nakagawa S, Morimoto N. Pediatric tracheostomy: Survival and longterm outcomes. Int J Pediatr Otorhinolaryngol 2016; 89: 81-5.

12. Mahadevan M, Barber $C$, Salkeld L, Douglas G, MıLıs N. Pediatric tracheotomy: 17 year review. 
Int J Pediatr Otorhinolaryngol 2007; 71: 182935.

13. Chittithavorn V, Rergkliang C, Chetpaophan A, VASINANUKORN P. Tracheo-innominate artery fistula in children with high-lying innominate artery. Asian Cardiovasc Thorac Ann 2006; 14: 514-6.

14. Brunettı M, Cohen J. Hematología. En: Manual Harriet Lane de pediatría. Robertson J y Shilkofski N editores. Editorial Elsevier Mosby, España 2006, capítulo 13.

15. Genther DJ, Thorne MC. Utility of routine postoperative chest radiography in pediatric tracheostomy. Int J Pediatr Otorhinolaryngol 2010; 74: 1397-400.

16. Yellon, R., Maguire, R., \& B. Tuchman, J. Pediatric tracheotomy. In P. Seidman, E. Sinz, \& D. Goldenberg (Eds.), Tracheotomy Management: A Multidisciplinary Approach. Cambridge: Cambridge University Press. 2011. doi:10.1017/ CB09780511977787.008.

17. White AC, Purcell E, Urouhart MB, Joseph B, O'Connor HH. Accidental decannulation following placement of a tracheostomy tube. Respir Care 2012; 57: 2019-25.

18. Mitchell RB, Hussey hM, Setzen G, Jacobs IN, Nussenbaum B, Dawson C, Brown CA 3rd, Brandt C, Deakins K, Hartnick C, Merati A. Clinical consensus statement: tracheostomy care. Otolaryngol Head Neck Surg 2013; 148: 6-20.

19. Weiss M, Knirsch W, Kretschmar 0, Dullenkopf $A$, Tomaske M, Balmer C, Stutz K, Gerber AC, Berger F. Tracheal tube-tip displacement in children during head-neck movement--a radiological assessment. Br J Anaesth 2006; 96: 486-91.

20. Furuya A, Nomura H, Kuroiwa G, Tamakı F, Suzuki S,
Nonaka A, Abe F, Matsukawa T. Endotracheal tube selection in children: which is the better predictor for the selection, tracheal internal diameter in $\mathrm{X}$-ray photograph or age-based formula. Masui 2009; 58: 724-7.

21. DeMauro S.B., Akinbi H., Maschroff K. Tracheostomy for the Neonate and Infant: Indications, Complications, Timing, and Outcomes. In: Lioy J., Sobol S. (eds) Disorders of the Neonatal Airway. Springer, New York, NY. 2015.

22. Wilcox LJ, Weber BC, Cunningham tD, BaldaSSARI CM. Tracheostomy Complications in Institutionalized Children with Long-term Tracheostomy and Ventilator Dependence. Otolaryngol Head Neck Surg 2016; 154: 725-30.

23. Monnier Ph. Acquired post- intubation and tracheostomy-related stenoses. En: capítulo 14: 183-98. Monnier Ph. Editor. Pediatric airway surgery. Management of laryngotracheal stenosis in infants and children.

24. Tejedor R, Antón-Pacheco Jl, Cabezalí D, López M, Villafruela MA, Gómez A. Surgical decannulation of children with suprastomal collapse: review of our experience. Cir Pediatr 2007; 20: 199-202.

25. Colman KL, Mandell DL, Simons JP. Impact of stoma maturation on pediatric tracheostomyrelated complications. Arch Otolaryngol Head Neck Surg 2010; 136: 471-4.

26. Lewis S, Arjomandi H, Rosenfeld R. Systematic review of surgery for persistent pediatric tracheocutaneous fistula. Laryngoscope 2017; 127: 241-6.

27. Cheng J, Setabutr D. Tracheocutaneous fistula closure in children. Int J Pediatr Otorhinolaryngol 2016; 89: 107-11.

\footnotetext{
Dirección: Hiram Álvarez Neri

Hospital Infantil de México, Federico Gómez

Dr. Márquez 162, Col. Doctores,

Delegación Cuauhtémoc, C.P. 06720, México, D.F.

Teléfono: 5228- 9917 extensión 2033

E mail: hiramhim@himfg.edu.mx / hiramhim@gmail.com
} 